\title{
$\boldsymbol{\beta}$-乳球蛋白在磁化水中的水合作用: 磁化处理对水分子缔合构造及 蛋白质水合特性的影响
}

\author{
和劲松 ${ }^{1,2, *}$ 杨宏伟 ${ }^{1, *}$ 蔡 然 ${ }^{1} \quad$ 罗之纲 $^{2}$ 祝万鹏 ${ }^{1}$ \\ (' 清华大学环境科学与工程系, 北京 $100084 ;{ }^{2}$ 康师傅控股有限公司中央研究所, 天津 300457)
}

\begin{abstract}
摘要：为探讨蛋白质在磁化水中的水合作用, 首先利用粘度测定及氧 17 核磁共振 $\left({ }^{17} \mathrm{O}-\mathrm{NMR}\right)$ 对经静磁场(MF) 处理不同有效时间 $\left(t_{\mathrm{eff}}\right)$ 后的纯水进行了分析, 进一步又利用差示扫描热量计(DSC)及 NMR 对溶解于磁化水的 $\beta$ 乳球蛋白 $(\beta-\mathrm{Lg})$ 的水合特性进行了分析. 随 $t_{\mathrm{eff}}$ 的增加, 水分子的内能不断减小, 处于氢键结合状态的水分子的比 例不断增加. 结果表明 MF 处理促进了水分子缔合结构的形成, 这一点可能与氢键的形成有关. 随 $t_{\mathrm{eff}}$ 的增加, $\beta$ $\operatorname{Lg}$ 表面水分子的运动性没有明显变化, 但 $\beta-\operatorname{Lg}$ 溶液中非自由结合水的含量不断增加. 说明 $\beta-\operatorname{Lg}$ 的水合作用与 水分子的缔合分布有关, 该分布依存于水分子的氢键状态并可通过磁场处理加以改变.
\end{abstract}

关键词：磁化；氢键；水分子缔合；蛋白质水合

中图分类号：0641

\section{Hydration of $\beta$-Lactoglobulin in Magnetized Water: Effect of Magnetic Treatment on the Cluster Structure of Water and Hydration Properties of Proteins}

\author{
HE Jin-Song ${ }^{1,2, *} \quad$ YANG Hong-Wei ${ }^{1, *} \quad$ CAI Ran ${ }^{1} \quad$ LO Chih-Kang ${ }^{2} \quad$ ZHU Wan-Peng ${ }^{1}$ \\ ('Department of Environmental Science and Engineering, Tsinghua University, Beijing 100084, P. R. China; \\ ${ }^{2}$ Central Research Institute, Ting Hsin International Group, Tianjin 300457, P. R. China)
}

\begin{abstract}
To investigate the hydration of proteins in magnetized water, pure water was treated with a static magnetic field (MF) over different effective treatment time $\left(t_{\text {eff }}\right)$. Viscosimetry and oxygen nuclear magnetic resonance $\left({ }^{17} \mathrm{O}-\mathrm{NMR}\right)$ spectra were recorded. The hydration properties of $\beta$-lactoglobulin $(\beta$ - $\mathrm{Lg})$ in magnetized water were examined by differential scanning calorimetry (DSC) and NMR. By increasing $t_{\text {eff }}$, the internal energy of the water decreased and the concentration of "hydrogen-bonded" water increased. This result indicates that MF treatment promotes the formation of water clusters, which can be attributed to an increase in hydrogen bonding. For the $\beta$-Lg solution prepared in magnetized water, the mobility of water molecules around the $\beta$ - $\mathrm{Lg}$ surface did not change significantly with an increase in $t_{\text {eff }}$ but the quantity of non-freezable bound water increased. This suggests that $\beta$-Lg hydration is related to the formation of water clusters, which depends on the hydrogen-bonding structure of water and can be varied by MF treatment.
\end{abstract}

Key Words: Magnetization; Hydrogen bond; Water molecule cluster; Protein hydration

Water is the most ubiquitous liquid on earth and is indispensable for life and the environment. Water has many unique properties such as large heat of vaporization, high boiling and melt- ing temperatures, and high solubility for charged and polar molecules ${ }^{[1]}$. The distinctive features of liquid water are mainly due to the three-dimensional hydrogen-bonding network formed

Received: August 9, 2009; Revised: October 28, 2009; Published on Web: December 9, 2009.

*Corresponding authors. Email: hejinsong@mail.tsinghua.edu.cn, yanghw@tsinghua.edu.cn; Tel: +86-10-62751724.

The project was supported by the National Natural Science Foundation of China (50608046).

国家自然科学基金(50608046)资助项目 
by water molecules ${ }^{[1-3]}$. Recently, changes in the structure of water under the effect of an external magnetic field (MF) were investigated, and it was observed that various aspects of the water structure changed upon exposure to an $\mathrm{MF}^{[4-6]}$. These investigations clearly indicated that the distribution of molecules and the transition probability of valence, bond, and inner-layer electrons varied, but the constitution of molecules and atoms was not altered $\mathrm{d}^{[2-5]}$.

Water is an extremely unique and essential component of all living organisms. A living cell is composed of approximately $80 \%$ water, and this solvent is the matrix and medium for the origin and operation of life. For biopolymers such as proteins, biological functions are only possible in the hydrated state. When a protein interacts with water molecules, the free energy landscape that governs the folding, structure, and stability of proteins is defined ${ }^{[7-10]}$. Moreover, functional processes mediated by proteins, such as binding, recognition, and catalysis, often involve specific interactions with individual water molecules ${ }^{[10,11]}$.

It is known that the water molecules form clusters through hydrogen bonding. The more stable cluster numbers are called magic numbers. The sequence of magic numbers carries essential information on the electronic and ionic structures of the cluster and, consequently, the water properties ${ }^{[12]}$. Indeed, liquid water can be regarded as a distribution of clusters with different magic numbers. If it is possible to modify the magic numbers or the distribution of the cluster, we may be able to find many new uses for water, particularly in biosystems, because the conformation and function of the biopolymer are related to the intrinsic properties of the solvent.

MF treatment is known to have an obvious effect on the intrinsic properties of liquid water. However, there has been limited research on the biological functions of biopolymers (e.g., proteins) after their hydration in magnetized water. In this study, the effect of MF treatment on pure water was investigated. Subsequently, the hydration properties of $\beta$-lactoglobulin $(\beta-\mathrm{Lg})$ in magnetized water were investigated. The results obtained are discussed in terms of the hydrogen-bonded structure of water.

\section{Materials and methods}

\subsection{Materials}

The water used in this study was ultrapure water produced in a Milli-Q Millipore System (Milli-Q, Advantage, A10, USA). It had a resistivity of approximately $18.2 \mathrm{M} \Omega \cdot \mathrm{cm}$ at $25{ }^{\circ} \mathrm{C}$. The total organic carbon and dissolved oxygen levels of the water were below $0.05 \mathrm{mg} \cdot \mathrm{L}^{-1}$ and $5.0 \mathrm{mg} \cdot \mathrm{L}^{-1}$, respectively. Bovine $\beta$-lactoglobulin (a mixture of the A and B variants, purity $=95 \%$ ) was purchased from Sigma-Aldrich (Shanghai, China) and used without further purification.

\subsection{MF treatment}

The static MF used in this study was generated by an adjustable permanent magnet (KC-70C, Jieling Magnetic \& Devices Co., Ltd., China). The permanent magnet was made of sintered $\mathrm{NdFeB}$, and the effective magnetic flux area was $26 \mathrm{~mm} \times 75$ $\mathrm{mm}$. The adjustable magnetic flux density ranged of (10-1000) $( \pm 10 \%) \mathrm{mT}$.

A certain volume of purified water was circulated in a close loop from a thermostated closed glass tank through perspex tubing (section area $s=0.07 \mathrm{~cm}^{2}$, total length $150 \mathrm{~cm}$ ) using a peristaltic pump. The flow rate was set approximately at $1 \mathrm{~m} \cdot \mathrm{s}^{-1}$. A part of the tubing $(l=7.5 \mathrm{~cm})$ was inserted between the polar pieces of the magnetic device, which was the length of the MF. The magnetic induction density was fixed at $0.50 \mathrm{~T}$ and measured by a digital teslameter (HT100, Ningbo Bestway Magnet Co., Ltd., China).

In this configuration, the effective magnetic treatment time $t_{\text {eff }}$, which is the total time for which the water is exposed to the static MF, can be described as

$$
t_{\text {eff }}=\frac{s l}{V} t_{T}
$$

where $V$ is the volume of purified water and $t_{T}$ is the total treatment time.

\subsection{Viscosity measurements}

The viscosity of each water sample was measured using a viscometer (Physica MCR 300, Paar Physica, Germany) with the thermostat set at $25{ }^{\circ} \mathrm{C}$. A standard liquid was used to calibrate the viscometer. The sample cup was filled with $15 \mathrm{~mL}$ of the water sample, and the viscosity was measured with a double gap cylinder (DG 26.7).

\section{$1.4{ }^{17} \mathrm{O}-\mathrm{NMR}$ analysis}

All ${ }^{17} \mathrm{O}-\mathrm{NMR}$ experiments were conducted on a JNM-ECA600 superconductor spectrometer (JEOL, Tokyo, Japan). ${ }^{17} \mathrm{O}$ spectra were obtained at $81.4 \mathrm{MHz}$ using $90^{\circ}$ pulse $(11.8 \mu \mathrm{s})$. Each free induction decay (FID) measurement had 1024 scans with a recycle delay of $0.1 \mathrm{~s}$. The experimental temperature was maintained at $25{ }^{\circ} \mathrm{C}$.

\subsection{Spin-spin relaxation time $\left(T_{2}\right)$ measurements}

$T_{2}$ measurements of protons were carried out in an AV-400 Pulsed NMR spectrometer (Brucker, Billerica, MA, USA). A sealed capillary tube with $\mathrm{D}_{2} \mathrm{O}$, a locking substance, was inserted at the center of a 5-mm sample tube. The Carr-Purcel-MeiboomGill sequences were employed to measure $T_{2}$. The pulse width, repetition delay, variable delay between pulse and acquisition, and dwell time between data were $14.0 \mu \mathrm{s}, 4 \mathrm{~s}, 4.5 \mu \mathrm{s}$, and 62.5 $\mu \mathrm{s}$, respectively. Eight scans were acquired for each measurement.

\subsection{Differential scanning calorimetry (DSC)}

DSC measures the heat (enthalpy) absorbed or emitted by a sample subjected to linearly scanned temperatures. The temperature difference $\Delta T$ between the reference and sample is strictly proportional to the heat capacity of the sample and the heating rate and can be represented as

$$
\Delta T=\frac{m c_{p}}{D} q
$$

where $m$ is the mass of the sample, $c_{p}$ is its specific heat, $q$ is the linear heating rate, and $D$ is a constant defined by the construction of the DSC cell. The temperature difference $\Delta T$ is proportional to the plotted heat flow. The enthalpy change $\Delta H$ in the 
time interval $\left(t_{1}-t_{2}\right)$ can be obtained from the recorded temperature difference $\Delta T$ and is described by the general formula

$$
\Delta H=D \int_{t_{1}}^{t_{2}} \Delta T \mathrm{~d} t
$$

In a multifraction system such as a protein solution maintained at a temperature below $0{ }^{\circ} \mathrm{C}$, similar terms can be written for each fraction, namely, for ice, nonfreezable bound water, and bulk or freezable water ${ }^{[13,14]}$.

The melting temperatures of water in the sample were determined from the temperature at the maximum point of the corresponding enthalpy peaks. The mass of freezable water $W_{\mathrm{c}}$ was obtained by the equation

$$
W_{\mathrm{c}}=\frac{Q}{\Delta H}
$$

where $\Delta H$ is the melting enthalpy of this type of water, which is assumed to be the same as that of bulk water, and $Q$ is the heat absorbed during the melting process. $Q$ was calculated from the area of the endothermic peak ${ }^{[15]}$.

The quantity of nonfreezable bound water $W_{\text {nc }}$ was obtained from the difference between the mass of total water $W_{\mathrm{t}}$ and freezable water as follows:

$$
W_{\text {nc }}=W_{\mathrm{t}}-W_{\mathrm{c}}
$$

The DSC measurements were carried out at a heating rate of $q=5 \mathrm{~K} \cdot \mathrm{min}^{-1}$ on a heat-flux DSC system (DSC821, Mettler Toledo, Suisse). Prior to the experiment, the samples were cooled at the same rate of $q=5 \mathrm{~K} \cdot \mathrm{min}^{-1}$.

\subsection{Statistical analyses}

Statistical analyses were performed using the SPSS Version 11 package for Mac (SPSS Inc., USA). To visualize the results from multiple experiments, the data from the replicates were subjected to one-way ANOVA and Duncan's multiple range tests, and a significance level of 0.05 was used.

\section{Results}

\subsection{Effect of MF treatment on water properties}

\subsubsection{Viscosity analysis}

Liquid viscosity arises from intermolecular forces and the ability of thermal fluctuations, aided by an applied shear stress, to produce flow in the direction of shear ${ }^{[16]}$. The Eyring viscosity model is valuable for inspecting the internal energy changes of a pure liquid by viscosity analysis.

According to the Eyring theory ${ }^{[17]}$, the viscosity $(\eta)$ of a pure liquid is given by the equation

$$
\eta=\frac{h N_{\mathrm{A}}}{V_{\mathrm{m}}} \exp \left(\frac{E^{\prime}}{R T}\right)
$$

where $N_{\mathrm{A}}$ is Avogadro's number, $h$ is Planck constant, $V_{\mathrm{m}}$ is the volume per mole of liquid, $R$ is gas constant, $T$ is the absolute temperature, and $E^{\prime}$ is the molar activation energy for surmounting the energy barrier.

The energy barrier $E^{\prime}$ may also be regarded as the energy required to create a hole in the liquid that is large enough to receive a molecule ${ }^{[16]}$. Therefore, the change in the molar internal energy $\Delta E$ of the pure liquid can be represented as

$$
\Delta E=-\Delta E^{\prime}=-\left(E^{\prime}-E_{0}^{\prime}\right)
$$

where $E^{\prime}$ and $E_{0}^{\prime}$ are the molar activation energies of pure liquids with viscosities $\eta$ and $\eta_{0}$, respectively. Using Eqs. 6 and 7, the internal energy variation of a pure liquid, expressed as $\Delta E / R T$, can be calculated from the viscosity of a pure liquid as

$$
\frac{\Delta E}{R T}=\ln \left(\frac{\eta}{\eta_{0}}\right)
$$

To investigate the effect of MF treatment on the properties of purified water, we determined the viscosity of purified water treated with a $0.5 \mathrm{~T}$ MF for different $t_{\text {effr }}$ The viscosity of MFtreated water increased with increasing $t_{\text {eff }}$ and had an amplification of approximately $10 \%$ when the $t_{\text {eff }}$ was 13 min (Fig.1). In addition, the value of $\Delta E / R T$ was calculated according to Eq.(8). In this case, $E_{0}^{\prime}$ and $\eta_{0}$ represent the molar activation energy and viscosity of the nontreated water, respectively. As shown in Fig. $1, \Delta E / R T$ decreased with increasing $t_{\text {eff. }}$ In other words, MF treatment diminished the molar internal energy of purified water, suggesting an increase in the molecular interactions of water.

\subsubsection{Measurement of the 170-NMR spectra}

With regard to the equilibrium and kinetic mechanisms of water molecules in the "free", "lattice", or "hydrogen-bonded" state, Hindman et al. ${ }^{[18]}$ have proposed that the mechanisms can be formally treated by starting with a two-phase model and examining the effect on the relaxation rate at different relative rates of exchange and molecular rotation. The equilibrium can be represented as

$$
\mathrm{H}_{2} \mathrm{O} \text { ("lattice”) } \underset{k_{1}}{\stackrel{k_{2}}{\rightleftharpoons}} \mathrm{H}_{2} \mathrm{O} \text { ("free”) }
$$

Moreover, $K=k_{2} / k_{1}$

$$
=\left[\mathrm{H}_{2} \mathrm{O} \text { ("free”)]/[ } \mathrm{H}_{2} \mathrm{O} \text { ("lattice”) }\right]
$$

and $k_{2}$ can be defined as the rotation rate of a "free" water molecule, and $k_{1}$ can be regarded as the rotation rate of a water molecule in a "lattice" site. In the case where the rate of chemical or phase exchange is fast relative to the rotation rate ${ }^{[18-19]}$,

$$
\begin{aligned}
& \frac{1}{T_{1}} \propto\left[\frac{1}{k_{2}}+\frac{1}{k_{2} K}\right] \\
& C=K /(1+K)
\end{aligned}
$$

where $C$ is concentration of "free" water. In addition, the ${ }^{17} \mathrm{O}$ resonance in water is much broader than the proton resonance ${ }^{[20]}$. The main relaxation mechanism is provided by the coupling of the quadrupole moment of the nucleus with electrical field gra-

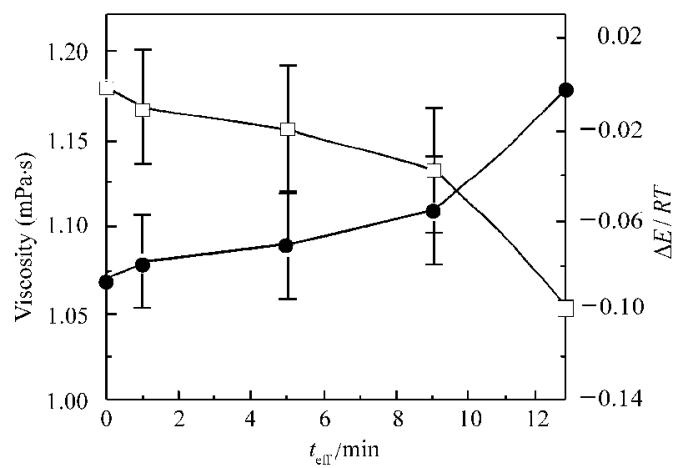

Fig.1 Viscosity $(\bullet)$ and internal energy change $(\Delta E / R T, \square)$ of pure water treated at $0.5 \mathrm{~T}$ for different $t_{\text {eff }}$ Bars show the standard deviation. 
dients. For a Lorentzian line, the full width at half-amplitude $\Delta \nu_{1 / 2}$ is related to the spin-spin relaxation time $T_{2}$ and the spinlattice relaxation time $T_{1}$ by the following equation ${ }^{[21,22]}$

$$
\Delta \nu_{1 / 2}=\frac{1}{\pi T_{2}} \propto \frac{1}{T_{1}}
$$

Using Eqs. $(11,13)$, the variation of the concentration of "lattice" water expressed as $\Delta C / C_{0}=\left(C-C_{0}\right) / C_{0}$ can be represented as

$$
\frac{\Delta C}{C_{0}}=\frac{\Delta \nu_{1 / 2}^{0}-\Delta \nu_{1 / 2}}{\Delta \nu_{1 / 2}}
$$

where $C$ and $C_{0}$ are the concentrations of "free" water in pure water with half-peak widths of $\Delta \nu_{1 / 2}$ and $\Delta \nu_{1 / 2}^{0}$, respectively. In this study, $\Delta C / C_{0}$ was used as a quantitative index for describing the transition of water molecules from a "free" state to a "lattice" site. In the "lattice" site, the monomeric water molecules form a cluster structure by hydrogen bonding.

To study the effect of MF treatment on the formation of water clusters, a ${ }^{17} \mathrm{O}$ NMR experiment was performed in which water was treated with an MF of $0.5 \mathrm{~T}$. The values of $\Delta C / C_{0}$ with different $t_{\mathrm{eff}}$ values were calculated according to Eq.(14). In this case, $C_{0}$ and $\Delta \nu_{1 / 2}$ are the concentrations of "free" water and the halfamplitude width of nontreated water, respectively. As shown in Fig.2, the $\Delta \nu_{1 / 2}$ value of purified water did not change when $t_{\text {eff }}$ was $1 \mathrm{~min}$ but increased with an increase in $t_{\text {eff }}$ when $t_{\text {eff }}>1$ min. On the other hand, as the $t_{\text {eff }}$ increased, the $\Delta C / C_{0}$ decreased, i.e., the concentration of the "hydrogen-bonded" water increased (Fig. 2). These results indicated that MF treatment promoted the formation of water clusters and altered their distribution such that larger clusters were formed. This could be attributed to an increase in the intermolecular interactions of MF-treated water (Fig. 1).

\subsection{Protein hydration in MF-treated water \\ 2.2.1 DSC analysis}

To determine the relationship between the formation of water clusters and the hydration properties of a protein, $\beta$-Lg solutions $\left(25 \mathrm{mg} \cdot \mathrm{mL}^{-1}\right)$ were prepared using MF-treated water with different $t_{\mathrm{eff}}$ values, and they were analyzed by DSC.

Fig. 3 shows a series of typical ice-melting DSC curves for $\beta$ Lg solutions prepared with MF-treated water. Approximately at

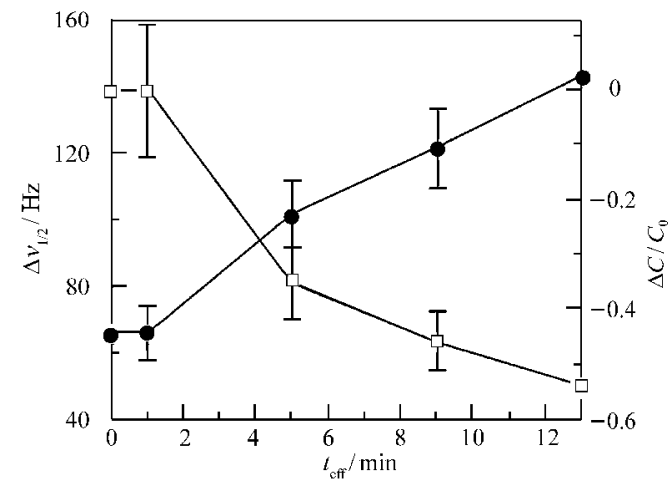

Fig.2 Half-peak width of ${ }^{17} \mathrm{O}-\mathrm{NMR}\left(\Delta \nu_{1 / 2}, \bullet\right)$ and concentration of "free" water $\left(\Delta C / C_{0}, \square\right)$ in pure water treated at $0.5 \mathrm{~T}$ for different $t_{\text {eff }}$

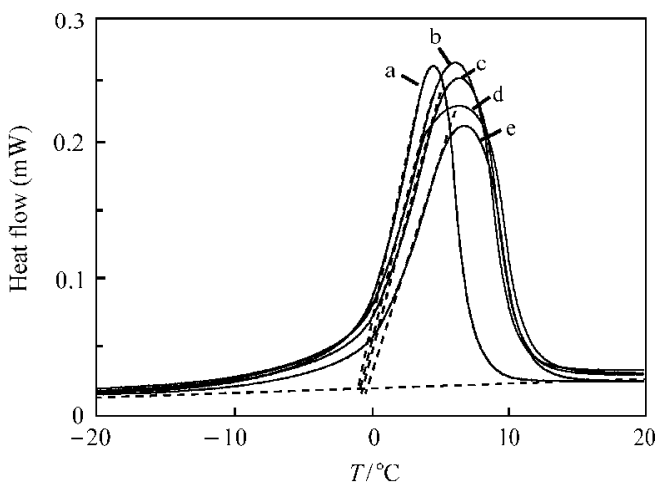

Fig.3 DSC curves measured during the ice-water phase transition of the $\boldsymbol{\beta}$ - $\mathrm{Lg}$ solution

The $\beta$ - $\mathrm{Lg}$ solution ( $25 \mathrm{mg} \cdot \mathrm{mL}^{-1}$ ) was prepared in pure water treated at $0.5 \mathrm{~T}$ for $t_{\text {eff }}$ of $0 \min ($ a), $1 \mathrm{~min}$ (b), $5 \mathrm{~min}$ (c), $9 \mathrm{~min}$ (d), and $13 \min (\mathrm{e})$.

$0{ }^{\circ} \mathrm{C}$, water exhibits a rapid increase in the heat flow that reflects the melting. When melting has completed, the heat flow returns exponentially to the level determined by the heat capacity of the liquid. This is a transient effect that indicates how the sample temperature increases from the inital state to the stationary state of any transition.

The initial melting temperature $\left(T_{\mathrm{m}}\right)$ was determined from the intersection of the linear portions of the melting curves drawn in the domain of initial state and transition state (Fig.3). A longer $t_{\text {eff }}$ results in a higher $T_{\mathrm{m}}$. In addition, the $\beta$ - $\mathrm{Lg}$ solution shows a small shift in the enthalpy peak toward higher temperatures and a decrease in the area of the enthalpy peak with increasing $t_{\text {eff }}$. These results indicate that changes in the clustering of water can alter the hydration properties of proteins. Furthermore, the quantity of non-freezable bound water was calculated by Eqs. (4 and 5). As shown in Fig.4, the mass ratio of nonfreezable bound water to the protein $\left(w_{\text {bound }}\right)$ increased with increasing $t_{\text {eff }}$ (significance level $(P)<0.05)$, except for the sample with a $t_{\mathrm{eff}}$ of $1 \mathrm{~min}$. These results indicate that an increase in the cluster size of water could increase the quantity of bound water in the hydration layer of $\beta$ - Lg.

2.2.2 Spin-spin relaxation measurements

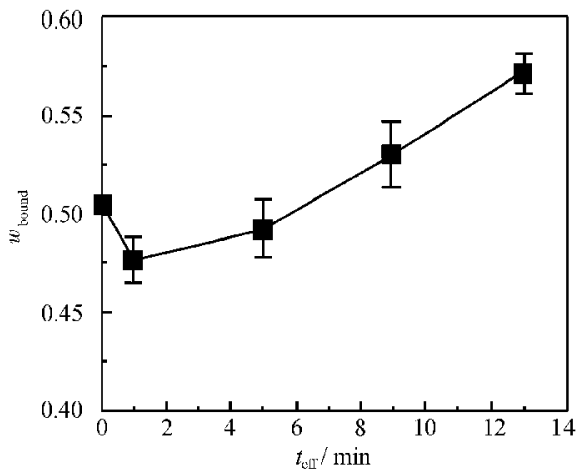

Fig.4 Variation in the quantity of nonfreezable bound water in the $\beta$-Lg solution as a function of $\boldsymbol{t}_{\text {eff }}$

The $\beta$ - $\mathrm{Lg}$ solution $\left(25 \mathrm{mg} \cdot \mathrm{mL}^{-1}\right)$ was prepared in pure water treated at $0.5 \mathrm{~T}$ for different $t_{\mathrm{eff}}$. 

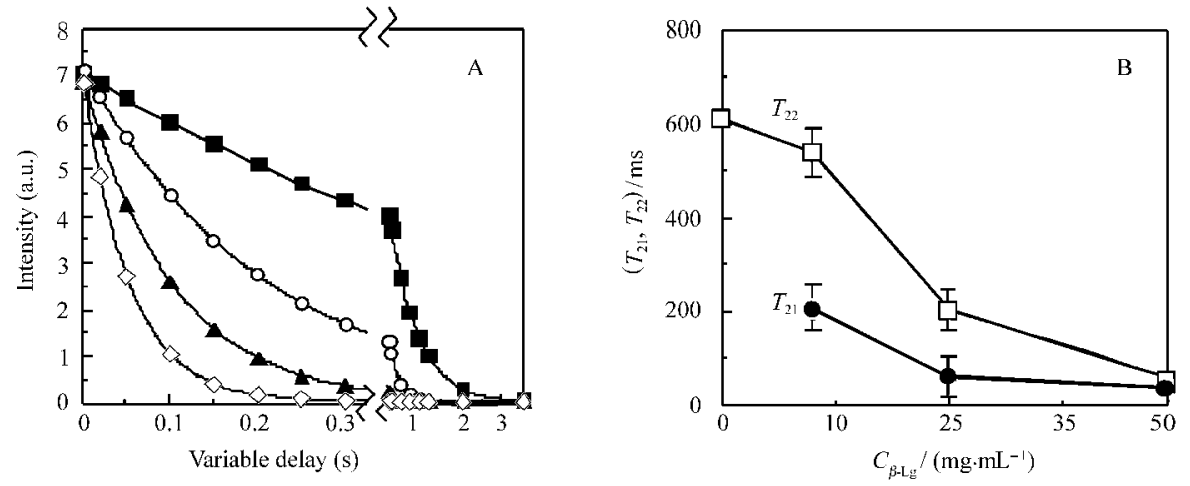

Fig.5 Plot of proton spin-spin relaxation decay (A) and relaxation time constant (B) of the $\beta$-Lg solution The $\beta$ - $\mathrm{Lg}$ solutions were prepared in pure water with a concentration of $0 \mathrm{mg} \cdot \mathrm{mL}^{-1}$

, $10 \mathrm{mg} \cdot \mathrm{mL}^{-1}(O), 25 \mathrm{mg} \cdot \mathrm{mL}^{-1}(\mathbf{\Lambda})$, and $50 \mathrm{mg} \cdot \mathrm{mL}^{-1}(\diamond)$.
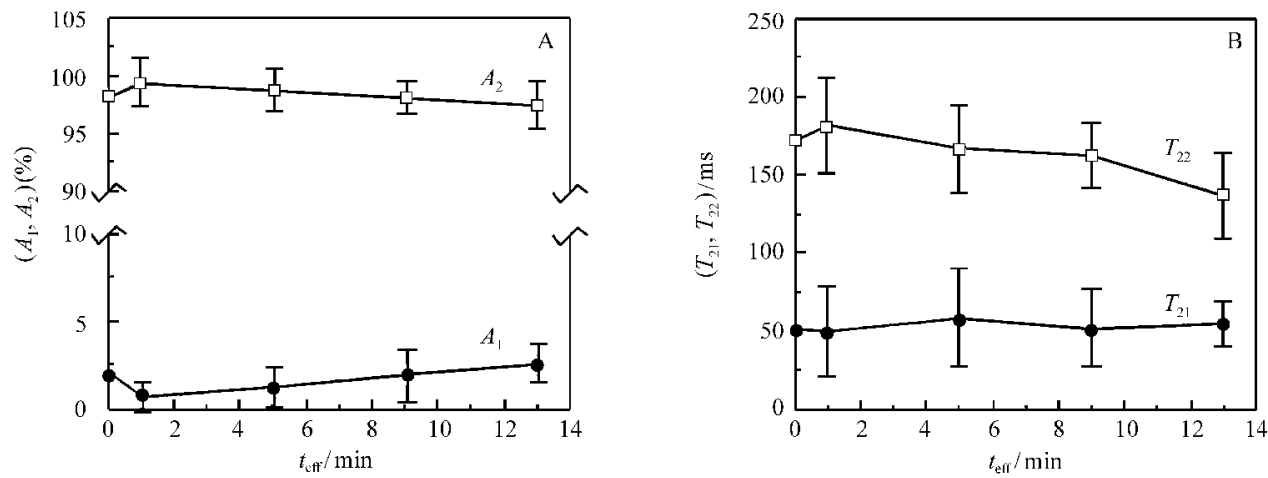

Fig.6 Variations in the proton intensity (A) and proton spin-spin relaxation time constant (B) of the $\beta$-Lg solution as a function of $\boldsymbol{t}_{\mathrm{ef}}$

The $\beta$ - $\mathrm{Lg}$ solution $\left(25 \mathrm{mg} \cdot \mathrm{mL}^{-1}\right)$ was prepared in pure water treated at $0.5 \mathrm{~T}$ for different $t_{\text {effi- }}$

As we know, NMR has been widely used in the studies of protein dynamics ${ }^{[23-24]}$. To clarify whether the changes in the clustering structure of water could also affect the mobility of the water molecules in the protein solution, ${ }^{1} \mathrm{H}$ NMR spin-spin relaxation measurements were carried out.

A typical plot of the spin-spin relaxation data of the biopolymerwater system does not generally follow a simple exponential decay $^{[25]}$ because more than one state of water is excited and the exchange between different states is slow in comparison to their relaxation times ${ }^{[26]}$. Therefore, the following two-component model was used:

$$
A=A_{1} \exp \left[-\frac{t}{T_{21}}\right]+A_{2} \exp \left[-\frac{t}{T_{22}}\right]
$$

where $A$ represents the proton intensity, which is proportional to the apparent water content of the sample. The two terms on the right-hand side of Eq. (15) represent two components of the relaxation curve; $A_{1}$ and $T_{21}$ are the proton intensity and spin-spin relaxation time constant of one component, respectively, while $A_{2}$ and $T_{22}$ are the corresponding values of the other component ${ }^{[25]}$. The component with the shorter relaxation time constant, i.e., $T_{21}$, corresponds to the protons in the less mobile fraction of water, and the component with the longer relaxation time constant, i.e., $T_{22}$, corresponds to the more mobile fraction of water in the sample ${ }^{[25-27]}$. However, it is necessary to note that the $T_{21}$ and $T_{22}$ values should be considered as averages for the water segment in each of the fractions because in each fraction, there may be molecules bound to different extents ${ }^{[26]}$.

In a protein solution, water molecules may exist in different states in which they have different mobilities ${ }^{[25,28-29]}$. As shown in Fig.5A, the FID curve of the $\beta$ - $\mathrm{Lg}$ solution (concentrations ranging from 10 to $50 \mathrm{mg} \cdot \mathrm{mL}^{-1}$ ) fitted fairly well when a two-component model was used (regression coefficient $\left(r^{2}\right)>0.99$ ). This indicated the presence of two fractions of water that have different relaxation rates or degrees of mobility, and $T_{2}$ could be represented by two fractions $T_{21}$ and $T_{22}$, as shown in the Eq.(15). $T_{21}$ and $T_{22}$ decreased with an increase in the concentration of $\beta$ - $\mathrm{Lg}$ (Fig.5B) and differed significantly when the concentration of $\beta$ $\mathrm{Lg}$ was below $25 \mathrm{mg} \cdot \mathrm{mL}^{-1}(P<0.05)$.

Consequently, a $\beta$ - $\mathrm{Lg}$ solution of $25 \mathrm{mg} \cdot \mathrm{mL}^{-1}$ was prepared using MF-treated water with different $t_{\text {eff. }}$ The FID curve of these $\beta$-Lg solutions also fitted fairly well when a two-component model was used $\left(r^{2}>0.99\right)$ (data not shown). As shown in Fig.6, $A_{2}$ and $T_{21}$ were almost constant; however, $T_{22}$ decreased and $A_{1}$ trended to increase with an increase in $t_{\text {eff }}$. These results indicated that enhancing the formation of water clusters would decrease the mobility of bulk water but would not have an obvious effect on the mobility of water molecules around the protein surface. 


\section{Discussion}

It was confirmed that water could be magnetized if it is exposed to an external $\mathrm{MF}^{[30-32]}$. The viscosity increased when the water was treated with an MF of $0.5 \mathrm{~T}$ (Fig.1). The same tendency was observed for the surface tension and vaporization enthalpy of water ${ }^{[31-33]}$. In addition, the melting temperature of water subjected to an MF of $6 \mathrm{~T}$ was $5.6 \mathrm{mK}$ higher than that of water that had not been treated with an $\mathrm{MF}^{[34]}$. An increase in the values of these physicochemical parameters indicates an increase in the molecular interactions of water.

Chang and Weng ${ }^{[5]}$ investigated the effects of an MF on the hydrogen-bonded structure of water and found that the number of hydrogen bonds increased by approximately $0.34 \%$ when the MF strength increased from 1 to $10 \mathrm{~T}$. This is consistent with the findings of Hosoda et al. ${ }^{[3]}$ who suggested that the enhancement in the hydrogen-bonded strength under an MF of $10 \mathrm{~T}$ is caused by increased electron delocalization in the hydrogen-bonded molecules. In addition, Iwasaka and Ueno ${ }^{[2]}$ found that the nearinfrared spectrum of water shifted to the low-frequency side when an MF of $14 \mathrm{~T}$ was applied. This effect is believed to be due to the strengthening of the hydrogen-bonding interactions by the application of the MF. The effect of the MF for increasing the number of hydrogen bonds is consistent with our results in which the internal energy of water decreases after MF treatment (Fig.1). This is because as more hydrogen bonds are formed in water, the water molecules becomes more inactive, which results in reduced internal energy of water. Indeed, Zhou et al. ${ }^{[35]}$ have investigated the influence of an external MF on the internal energy of pure water using Monte Carlo simulations and concluded that when the MF strength is above $0.23 \mathrm{~T}$, the internal energy decreases in comparison with the field-free case.

Water clusters have equal hydrogen bonding in all directions. A higher number of hydrogen bonds implies that the size of the water cluster has increased. Since the concentration of the "lattice" water in the magnetized water increased in comparison with that of nontreated water (Fig.2), this result indicates that the application of an MF leads to changes in the assembly and distribution of water clusters. Thus, clusters with different forms of water chains formed by hydrogen bonding appear in the system.

It is necessary to note that the cyclic magnetization treatment used in this study is different from the static magnetization treatment, the magnetization effect of former was dependent on the treatment time (Figs. $(1,2)$ ), but not for the latter (data not shown). As a result, the influence of static MF of NMR equipment on the sample is weaker than that of cyclic magnetization treatment, and could be ignored.

We also analyzed the hydration of $\beta$ - $\mathrm{Lg}$ in magnetized water (Figs. $(3,4,6)$ ). In general, water present in a protein solution can be of three types: "free water", which freezes at the usual freezing point; "intermediate water", which freezes at a temperature lower than the usual freezing point; and "unfrozen bound water", which cannot freeze at the usual freezing point ${ }^{[14]}$. In this three-state water model, it is believed that the bound water is generated and becomes unfrozen presumably due to the formation of hydrogen bonds between water and polar groups on the polymer ${ }^{[36-38]}$. At approximately $0{ }^{\circ} \mathrm{C}$, a peak of melting ice is observed on the DSC thermogram of the $\beta$-Lg solution (Fig.3). The quantity of freezable water was lower than the total water quantity in the protein solution. The rest of the water, i.e., the nonfreezable water, corresponds to the water molecules that interact with the protein ${ }^{[15]}$. This interaction would consist of well-oriented hydrogen bonds between water (H-bond donor) and the polar groups (H-bond acceptor) ${ }^{[29]}$. When the hydrogen-bonded structure of water was enhanced after MF treatment (Figs. $(1,2)$ ), the water molecules could easily interact with the protein through hydrogen bonds due to the lower mobility of the water molecules (Figs.(1, 6)). This would extend the lifetime of the water molecules in the hydration layer and result in an increase in the quantity of nonfreezable bound water in the $\beta$-Lg solution (Fig.4).

Our results also showed that fractions containing water molecules with both fast and slow dynamics coexisted in the $\beta$ Lg solution (Figs. $(5,6)$ ). Near the surface of the protein, the hydrogen-bonded structure of water became locally modified. Therefore, the structure and properties of the hydration water near the surface differed greatly from those of bulk liquid wa$\operatorname{ter}^{[11,39]}$. As a result, while water molecules near the protein were certainly slow, the hydration layer became dynamically rigid (Figs. $(5,6)$ ). The dynamics of water in the hydration layer is partly determined by the relaxation kinetics of protons, which in turn depends on the structure of the layer and the activation energy of the water molecules ${ }^{[39]}$. When $\beta$ - Lg was hydrated in magnetized water, although the fraction of water molecules $\left(A_{1}\right)$ with low dynamics $\left(T_{21}\right)$ tended to increase with increasing $t_{\text {eff }}$ (Fig.6A), the mobility of this fraction $\left(T_{21}\right)$ did not change significantly (Fig.6B). This suggests that the dynamics of water around the protein surface mainly depends on the structure of the hydration layer induced by the protein surface. An increase in the quantity of nonfreezable bound water ( or $A_{1}$ ) may occur due to an increase in the hydrogen bonding of the fraction of water called "intermediate water."

Finally, understanding the effects of MF treatment on the properties of water and hydration of proteins may provide information that can be practically applied. Further studies on the conformation of proteins in magnetized water are important for clarifying the effects of the water clustering structure on biosystems. In addition, it would be important to examine the timedependent change of water after treatment by MF, and to study the effect of cyclic magnetization treatment on the water using the low-field NMR equipment. Such studies are currently underway.

\section{Conclusions}

After treatment with a static MF, the molar internal energy of pure water was diminished, and the distribution of water clusters shifted such that larger clusters were formed. These results can 
be attributed to the promotion of the formation of the hydrogenbonded structure of water induced by MF treatment. When $\beta$-Lg was hydrated in magnetized water, the mobility of water molecules on the $\beta$-Lg surface did not change significantly, but the quantity of nonfreezable bound water increased with an increase in the treatment time. This may be due to changes in the distribution of the water clusters, which in turn depends on the hydrogen-bonded structure of water.

\section{References}

1 Eisenberg, D.; Kauzmann, W. The structure and the properties of water. Oxford: Oxford University Press, 1969: 32-108

2 Iwasaka, M.; Ueno, S. J. Appl. Phys., 1998, 83: 6459

3 Hosoda, H.; Mori, H.; Sogoshi, N.; Nagasawa, A.; Nakabayashi, S. J. Phys. Chem. A, 2004, 108: 1461

$4 \quad$ Pang, X.; Deng, B. Physica B, 2008, 403: 3571

5 Chang, K. T.; Weng, C. I. J. Appl. Phys., 2006, 100: 043917

6 Deng, B.; Pang, X. F. Chin. Sci. Bull., 2007, 52: 3179

7 Kauzmann, W. Adv. Prot. Chem., 1959, 14: 1

8 Tanford, C. The hydrophobic effect: formation of micelles and biological membranes. New York: Johe Wiley, 1973: 42-101

9 Westhof, E. Annu. Rev. Biophys. Biophys. Chem., 1988, 17: 125

10 Saenger, W. Annu. Rev. Biophys. Biophys. Chem., 1987, 16: 93

11 Raschke, T. M. Curr. Opin. Struct. Biol., 2006, 16: 152

12 Kiselev, M.; Heinzinger, K. J. Chem. Phys., 1996, 105: 650

13 Tompa, P.; Bánki, P.; Bokor, M.; Kamasa, P.; Kovács, D.; Lasanda, G.; Tompa, K. Biophys. J., 2006, 91: 2243

14 Liu, W. G.; Yao, K. D. Polymer, 2001, 42: 3943

15 Ping, Z. H.; Nguyen, Q. T.; Chen, S. M.; Zhou, J. Q.; Ding, Y. D. Polymer, 2001, 42: 8461

16 Tabor, D. Gases, liquids, and solids: and other states of matter. 3rd ed. London: Cambridge University Press, 1991: 304-307

17 Eyring, H. J. Chem. Phys., 1936, 4: 283

18 Hindman, J. C.; Zielen, A. J.; Svirmickas, A.; Wood, M. J. Chem. Phys., 1971, 54: 621
19 Hindman, J. C. J. Chem. Phys., 1974, 60: 4488

20 Pessen, H.; Kumosinski, T. F. Methods Enzymol., 1985, 117: 219

21 Farrar, T. C.; Becker, E. D. Pulse and Fourier transform NMR: introduction to theory and methods. New York: Academic Press, 1971: 46-65

22 Meiboom, S. J. Chem. Phys., 1961, 34: 375

23 Peng, L. M.; Guo, X. F.; Ding, W. P. Chin. J. Magn. Reson., 2009, 26: 173 [彭路明, 郭学锋, 丁维平. 波谱学杂志, 2009, 26: 173]

24 Hu, Y. F.; Jin, C. W. Chin. J. Magn. Reson., 2009, 26: 151 [胡蕴菲, 金长文. 波谱学杂志, 2009, 26: 151]

25 Ruan, R. R.; Chen, P. L. Water in foods and biological materials: a nuclear magnetic resonance approach. Lancaster: CRC Press, 1997: $51-71$

26 Kuo, M. I.; Gunasekaran, S.; Johnson, M.; Chen, C. J. Dairy Sci., 2001, 84: 1950

27 Ruan, R. R.; Zou, C.; Wadhawan, C.; Martinez, B.; Chen, P. L.; Addis, P. J. Food Process. Preserv., 1997, 21: 91

28 Yang, P. H.; Rupley, J. A. Biochemistry, 1979, 18: 2654

29 Franks, F. Characterization of proteins. New York: Humana Press, 1988: 127-154

30 Holysz, L.; Szczes, A.; Chibowski, E. J. Colloid Interface Sci., 2007, 316: 996

31 Fujimura, Y.; Iino, M. J. Phys. Conf., 2009, 156: 012028

32 Ghauri, S. A.; Ansari, M. S. J. Appl. Phys., 2006, 100: 066101

33 Toledo, E. J. L.; Ramalho, T. C.; Magriotis, Z. M. J. Mol. Struct., 2008, 888: 409

34 Inaba, H.; Saitou, T.; Tozaki, K. -I.; Hayashi, H. J. Appl. Phys., 2004, 96: 6127

35 Zhou, K. X.; Lu, G. W.; Zhou, Q. C.; Song, J. H.; Jiang, S. T.; Xia, H. R. J. Appl. Phys., 2000, 88: 1802

36 Pei, Y.; Chen, J.; Yang, L.; Shi, L.; Tao, Q.; Hui, B.; Li, J. J. Biomater. Sci. Polym. Ed., 2004, 15: 585

37 Shimao, M. Curr. Opin. Biotechnol., 2001, 12: 242

38 Vert, M. Biomacromolecules, 2005, 6: 538

39 Bagchi, B. Chem. Rev., 2005, 105: 3197 